\title{
On découvre un lien entre un groupe de cas de tuberculose et le tabagisme : un défi sous-estimé dans l'élimination de la tuberculose
}

\author{
E Rea ${ }^{1,2 *}$, T Leung $^{1}$
}

\section{Résumé}

Contexte : Bien qu'on sache que le tabagisme augmente les risques d'infection à la tuberculose, de tuberculose active, de rechute après le traitement et de décès, on sous-estime fréquemment l'importance du rôle qu'il joue. En effet, il constitue au Canada un facteur de risque potentiellement réversible pour la santé publique et la pratique clinique.

Objectif : Examiner les données probantes actuelles sur le lien entre tabagisme et risques de contracter la tuberculose, décrire l'enquête menée sur un groupe de cas de transmission locale associée au tabagisme à Toronto et discuter des répercussions pratiques du tabagisme sur I'élimination de la tuberculose au Canada.

Enquête et intervention en santé publique : On a constaté trois cas de tuberculose dans le même lieu de travail sur une période de deux ans. Selon le génotypage, ces trois souches étaient identiques. Des entretiens poussés avec les personnes atteintes, leurs gestionnaires et les gérants de l'immeuble ont permis de confirmer qu'elles ne travaillaient pas ensemble et ne se fréquentaient pas. Le seul lien épidémiologique qu'on a observé, c'est qu'elles étaient des fumeurs réguliers qui fumaient dehors au même endroit. L'évaluation de la ventilation a confirmé que l'immeuble ne recyclait pas l'air non filtré entre les étages. À la lumière des données épidémiologiques et des tests en laboratoire, nous avons déterminé que l'endroit où la maladie s'est transmise est probablement la zone fumeurs partiellement recouverte, qui se trouve à l'extérieur du lieu de travail. Nous avons ouvert dans l'immeuble une clinique de dépistage active, dont nous avons annoncé l'existence à tous les travailleurs qui fréquentaient ces zones fumeurs. Chez les 60 personnes ayant passé le test de Mantoux, on n'a diagnostiqué aucun autre cas de tuberculose active. Une personne née au Canada a obtenu des résultats positifs à ce test. Enfin, nous avons offert des séances de sensibilisation à la tuberculose au personnel travaillant dans cet immeuble et nous en avons profité pour faire la promotion du renoncement au tabac auprès des personnes intéressées.

Conclusion : Ce groupe démontre que la tuberculose peut se transmettre par le tabagisme à Toronto. L'Organisation mondiale de la Santé fait la promotion de l'intégration de la lutte contre le tabagisme, qui constitue selon elle une stratégie importante d'élimination de la tuberculose. Parmi les moyens utilisés pour réaliser cette intégration au Canada, on compte l'évaluation de la transmission par le tabagisme au cours des enquêtes sur les contacts, le recours régulier à des outils de renoncement au tabac pour les contacts ou toute autre personne atteinte d'une infection latente ou active à la tuberculose, et une approche communautaire en matière de santé publique.

\author{
Affiliations \\ ${ }^{1}$ Bureau de santé publique de \\ Toronto, Ontario \\ 2 École de santé publique Dalla \\ Lana, Université de Toronto, \\ Toronto (Ontario)
}

*Correspondance : Elizabeth. Rea@toronto.ca

Citation proposée : Rea E, Leung T. On découvre un lien entre un groupe de cas de tuberculose et le tabagisme : un défi sous-estimé dans l'élimination de la tuberculose. Relevé des maladies transmissibles au Canada. 2018;44(3/4):96-100. https://doi.org/10.14745/ccdr.v44i34a03f

\section{Introduction}

On soupçonne depuis une centaine d'années que le tabagisme constitue un facteur de risque pour la tuberculose, hypothèse qu'on a démontrée au cours des dernières décennies (1-3). En effet, le tabagisme nuit à plusieurs mécanismes biologiques jouant un rôle important dans la défense contre l'infection initiale à la tuberculose et le développement ultérieur de la phase active, dont une mauvaise élimination des sécrétions des voies respiratoires, le mauvais fonctionnement des macrophages pulmonaires et des cellules porteuses de la molécule CD4, et une réduction de la production d'interféron gamma et du facteur de nécrose tumorale alpha (4-6). Le tabagisme est associé à un risque accru d'infection à la tuberculose, de tuberculose active, de rechute après le traitement et de décès (tableau 1). Ce risque accru a été documenté chez des populations et dans des milieux très divers, que ce soit pour le tabagisme actif ou le tabagisme passif (10-12). 
Tableau 1 : Résumé des données sur le lien existant entre le tabagisme et le risque accru d'être atteint de la tuberculose

\begin{tabular}{|l|l|}
\hline \multicolumn{1}{|c|}{ Risque accru } & \multicolumn{1}{c|}{ Données } \\
\hline Infection tuberculeuse (1) & $\begin{array}{l}\text { Rapport de cotes pondéré : 1,83, IC à } 95 \%, \\
1,49 \text { à } 2,23\end{array}$ \\
\hline Phase active (2) & Risque relatif : 2,29, IC à $95 \%, 1,93$ à 2,71 a \\
\hline Rechute après le traitement (8) & $\begin{array}{l}\text { Rapport de risque ajusté : } 2,04, \text { IC à } 95 \%, \\
1,22 \text { à } 3,41^{\mathrm{b}}\end{array}$ \\
\hline $\begin{array}{l}\text { Décès attribuable à la } \\
\text { tuberculose (9) }\end{array}$ & Risque relatif : 4,5, IC à $95 \%, 4$ à 5 \\
\hline $\begin{array}{l}\text { Abréviations: IC, intervalle de confiance } \\
\text { a Avec relation dose/effet (7) } \\
\text { bPour les personnes fumant plus de } 10 \text { cigarettes par jour, par rapport aux non-fumeurs }\end{array}$
\end{tabular}

Ces risques relatifs faibles - environ le double des risques vécus par les non-fumeurs - peuvent devenir des risques importants pour l'ensemble de la population. Selon l'Organisation mondiale de la Santé, le tabagisme causerait indirectement $20 \%$ des cas de tuberculose dans le monde (3). En effet, une étude d'envergure a calculé que la fraction étiologique du risque dont il est responsable atteindrait $38 \%$ des cas chez les Indiens de sexe masculin (13). Malgré ces données, nous savons par expérience qu'il est rare qu'on s'intéresse systématiquement au tabagisme lorsqu'on organise des activités de gestion de la tuberculose à l'échelle locale. Même si les Normes canadiennes pour la lutte antituberculeuse et les Orientations pour les programmes de prévention et de contrôle de la tuberculose au Canada déterminent que le tabagisme constitue un facteur de risque de contracter la tuberculose, aucun de ces deux documents ne propose de mesures antitabac explicites dans les recommandations qu'il émet sur les soins cliniques ou la prévention programmatique de cette maladie $(14,15)$.

Dans l'ensemble de la population canadienne, le taux de tabagisme est de $18 \%$ (16). II faut toutefois préciser que ce taux est bien plus élevé chez certains groupes davantage atteints par la tuberculose, dont les sans-abris et un grand nombre de villages autochtones $(17,18)$. Cela dit, le tabagisme est un problème pour toutes les personnes vulnérables à la tuberculose, et ce, quelle que soit la population à laquelle elles appartiennent.

À Toronto, on dénombre chaque année trois cents de cas de tuberculose active. Plus de $90 \%$ des personnes atteintes ne sont pas nées au Canada. À titre de comparaison, la moitié des Torontois ne sont pas nés au pays $(19,20)$. Nous décrivons ici un groupe de transmissions de la tuberculose associée au tabagisme au sein de la population torontoise, et nous proposons un programme de santé publique et ses incidences cliniques.

\section{Description du groupe}

Le premier cas était un homme de 34 ans né en Inde, qui s'est mis à manifester les symptômes de la tuberculose environ un an après être arrivé au pays. En juillet 2015, on a diagnostiqué chez cet homme, qui vivait seul et a avoué être un fumeur invétéré, une tuberculose cavitaire pulmonaire accompagnée d'un résultat au frottis de ses expectorations de 4+. On a trouvé deux contacts dans l'immeuble où il travaillait, mais on les a perdus pendant le suivi. Au cours des deux années suivantes, on a diagnostiqué la tuberculose chez deux autres travailleurs de cette grande tour comptant plusieurs bureaux différents, où travaillaient environ 1200 personnes.
Le deuxième cas était celui d'un homme de 55 ans, né au Canada, ayant peu voyagé et sans antécédents marquants d'exposition à la tuberculose. Lors d'un examen de diagnostic en décembre 2016, un frottis des expectorations a indiqué chez lui un résultat de $2+$ et sa radiographie indiquait une anomalie non cavitaire dans sa poitrine. Il a déclaré fumer chaque semaine deux paquets de cigarettes. II travaillait à un étage différent, mais dans le même immeuble que le premier cas, ne s'était pas lié d'amitié avec les autres travailleurs de cet immeuble et mangeait seul.

Le troisième cas était un homme de 52 ans né en Inde, qui a reçu son diagnostic en avril 2017. Il a obtenu un résultat au frottis de $1+$ lors du diagnostic et a déclaré fumer un paquet par semaine. Sa radiographie indiquait une anomalie non cavitaire dans sa poitrine. Il travaillait au même étage que le premier cas, mais dans un secteur différent. Ces trois personnes n'étaient pas les contacts des unes des autres.

\section{Résultats du génotypage}

Le génotypage par les unités répétitives dispersées sur le génome mycobactérien à 24 locus et le spoligotypage des trois isolats de tuberculose ont donné des résultats identiques. Le séquençage du génome entier des frottis indiquait, pour les cas $n^{\circ} 1$ et 2 , un polymorphisme nucléotidique sur un seul nucléotide; pour les cas $\mathrm{n}^{\circ} 1$ et 3 , sur cinq nucléotides; et, pour les cas $n^{\circ} 2$ et 3 , sur six nucléotides. Ces résultats laissent croire que le cas $n^{\circ} 1$ était le cas source des deux autres.

\section{Enquête et intervention en santé publique}

Au moment de l'enquête, le cas $n^{\circ} 1$ avait quitté le pays. Malgré ce contretemps, après avoir interrogé une deuxième fois les deux hommes restés au Canada, ainsi que leurs gestionnaires et les gérants de l'immeuble, on a réussi à découvrir que les trois hommes n'avait aucun lien social ou professionnel, mais qu'ils étaient tous des fumeurs réguliers. Ils fréquentaient tous la même zone fumeurs partiellement couverte, située près de l'entrée principale.

L'évaluation de la ventilation a confirmé que l'immeuble ne recyclait pas l'air non filtré entre les étages. Nous en déduisons qu'il est peu probable que la transmission se soit faite par la circulation de l'air d'une pièce à l'autre. À la lumière des données épidémiologiques et des tests en laboratoire, nous avons déterminé que les endroits où la maladie s'est transmise sont probablement les zones fumeurs qui se trouvent à l'extérieur du lieu de travail et que le cas $n^{\circ} 1$ aurait infecté les deux autres hommes.

Étant donné que la maladie s'est transmise entre fumeurs qui ne sont pas considérés comme des amis et qu'il n'existait aucune liste des contacts permettant d'identifier les personnes vulnérables, nous avons adopté une approche géographique pour assurer le suivi des contacts. Nous avons ouvert dans l'immeuble une clinique de dépistage active, dont nous avons annoncé l'existence à tous les travailleurs qui fréquentaient ces zones fumeurs (dépistage systématique de la phase active dans un groupe cible prédéterminé). Elle faisait passer des tests de Mantoux et prélevait les expectorations chez les fumeurs et les non-fumeurs qui fréquentaient les zones fumeurs situées près de l'immeuble entre le moment où le cas $n^{\circ} 1$ était contagieux jusqu'au moment de l'enquête. Chez les 60 personnes vues par cette clinique, nous n'avons diagnostiqué aucun autre cas de tuberculose active. Nous avons détecté une conversion chez une seule personne pour le test de Mantoux. Nous lui avons alors recommandé un traitement pour sa tuberculose latente. Enfin, 
nous avons offert des séances de sensibilisation à la tuberculose au personnel travaillant dans cet immeuble et nous en avons profité pour faire la promotion du renoncement au tabac auprès des personnes intéressées.

\section{Discussion}

Ce groupe indique de façon probante que la tuberculose se transmet par le tabagisme à Toronto : les trois hommes n'avaient aucun autre lien et ne faisaient pas partie de groupes marginalisés, même si le proposant est né dans un pays fortement touché par cette maladie. II est particulièrement déconcertant que la transmission se soit produite à l'extérieur.

D'après notre expérience, le tabagisme est souvent sous-estimé - et les mesures antitabac trop peu souvent appliquées - dans les programmes antituberculeux de santé publique et dans les soins de santé cliniques.

En termes pratiques, il existe quatre stratégies principales d'intégration du tabagisme et de la lutte antituberculeuse :

- évaluer le risque de transmission chez les fumeurs dans le cadre des enquêtes menées sur les contacts

- avoir comme méthode d'appoint l'incorporation systématique de mesures antitabac dans le but de prévenir la phase active de la tuberculose chez les contacts et toute personne atteinte de la forme latente

- améliorer les résultats cliniques chez les personnes atteintes de la phase active

- $\quad$ adopter une approche communautaire stratégique antitabac chez les populations très vulnérables à la tuberculose, en collaboration avec les programmes de prévention de la toxicomanie

On sait que le risque de transmission est plus élevé dans les endroits surpeuplés ou mal aérés (14). L'exemple le plus extrême de ce phénomène est le "hot-boxing ", où des consommateurs de tabac ou de drogue fument dans un espace fermé et restreint comme une voiture ou une petite pièce, afin de concentrer la fumée de marijuana ou de toute autre substance et d'en intensifier ainsi les effets. Plusieurs enquêtes sur les éclosions ont déterminé que le fait de fumer de la marijuana $(21,22)$ ou du crack (23) pose un risque de transmission.

Quoique certaines populations soient particulièrement vulnérables à la transmission de la tuberculose par le tabagisme, en raison de leur forte densité de cohabitation et de leur taux de tabagisme élevé, les observations que nous avons réalisées à Toronto prouvent que la tuberculose peut se déclarer même dans une métropole où le taux de tabagisme global est faible et même chez les personnes qui ne sont pas marginalisées. Le groupe décrite en détail ci-dessus n'est pas l'unique exemple récent de transmission de la tuberculose par le tabagisme dans cette ville. En effet, nous avons aussi documenté au cours des deux dernières années des cas de transmission confirmés par le génotype : un cas associé à un salon de narguilé, six cas associés au " hot-boxing » de tabac ou de marijuana (dont trois sont aussi des contacts familiaux) et trois cas liés au balcon pour fumeurs d'un refuge pour les sans-abris.

On considère généralement que les expositions extérieures constituent un risque très faible de transmission de la tuberculose (14). Notre étude de cas illustre qu'elle peut se transmettre même dehors, particulièrement dans les espaces restreints couverts d'un surplomb - le genre d'espaces extérieurs partiellement fermés où s'abritent un grand nombre de travailleurs qui fument. Les expositions courtes mais fréquentes pendant les pauses cigarette peuvent s'accumuler et constituer un risque substantiel pour les gens qui fument régulièrement ensemble en compagnie d'une personne infectée, même s'ils ne se rencontrent pas ailleurs.

Il peut être plus difficile d'assurer le suivi de contacts faisant partie de groupes spontanés de fumeurs, dont les membres ne s'inscrivent pas et où la participation est souvent fluide. Ces fumeurs n'y voient pas toujours leurs semblables comme des amis ou des relations. Au lieu de demander «Avec qui fumez-vous?", il serait peut-être plus utile de leur demander "Qui d'autre est présent lorsque vous fumez? ». Qui plus est, les consommateurs de tabac ou de marijuana peuvent se sentir réticents à déclarer que d'autres ou eux-mêmes participent à une activité qui est soit illégale soit mal vue par la société. II peut être utile de procéder à un dépistage géographique et d'adopter une approche créative qui soit adaptée à la situation. Bien que la décriminalisation de la marijuana (et la hausse éventuelle de sa consommation) au Canada en 2018 ne pose sans doute pas de risque que la majorité des Canadiens contractent la tuberculose, il serait sage néanmoins d'assurer le suivi de ses répercussions sur les communautés et les populations fortement touchées par cette maladie.

Les fumeurs ayant une tuberculose latente profiteront sans doute davantage d'un traitement contre la phase latente, car leur risque relatif de contracter la forme active est plus élevé que celui des non-fumeurs. Une attention plus explicite à ce facteur de risque dans les soins cliniques ou le counseling réguliers, notamment pour les contacts, peut contribuer à convaincre plus de patients atteints de la forme latente de suivre le traitement. De même, il serait utile, dans le cadre des soins réguliers prodigués aux personnes ayant la forme latente, de donner des séances de counseling antitabac aux fumeurs atteints de tuberculose latente ou de leur recommander de le faire, qu'ils aient décidé ou non de suivre le traitement. Les fumeurs en phase active risquent d'éprouver des maux plus graves et auraient également besoin de soutien pour les aider à cesser de fumer.

Sur le plan stratégique, comme c'est le cas pour les populations ayant des taux de diabète élevés, les programmes antituberculeux pourraient collaborer avec les programmes de prévention de la toxicomanie ou du tabagisme, en vue de lancer des initiatives de prévention et de cessation du tabagisme adaptées à la culture de groupes locaux souffrant de taux de tuberculose et de tabagisme plus élevés. On entend par là non seulement les Autochtones et les sans-abris, mais aussi certains groupes d'immigrants. À l'échelle nationale, c'est l'important bassin de personnes atteintes de la forme latente - qui sont surtout des patients nés à l'étranger - qui constituent l'essentiel des gens souffrant de tuberculose au Canada (24). De plus, notre groupe souligne l'importance de règlements locaux et de mesures assurant la santé et la sécurité au travail. En effet, si un grand nombre d'employeurs interdisent à leurs employés de fumer à proximité des entrées, nous ne savons pas s'il existe des interdictions de fumer dans les abris partiellement fermés situés près du lieu de travail.

Ce qui plus important encore, le Canada sous-estime peut-être l'effet accélérateur du tabagisme dans la transmission de la tuberculose, surtout dans les communautés les plus touchées par les éclosions : sans-abris, réserves autochtones du Nord et villages inuit. Dans ces collectivités, les hauts taux d'incidence de la tuberculose, la forte prévalence du tabagisme et la densité élevée de cohabitation pourraient causer une explosion de la transmission. L'Enquête auprès des peuples 
autochtones de 2012 a révélé que 52 \% des Inuit âgés de 15 ans et plus fumaient des cigarettes tous les jours, soit près de trois fois le taux global canadien (25). Le Nunavut affiche aussi le taux le plus élevé de tuberculose active au Canada, soit 170 cas pour 100000 habitants en 2016. Ce taux est plus que 290 fois plus élevé que le taux de tuberculose active chez les non-Autochtones nés au Canada (26). Étant donné que le risque le plus élevé de contracter la tuberculose active se situe dans les deux premières années suivant l'infection (14), dans les situations où le tabagisme est répandu, le taux plus élevé à la fois de transmission de la tuberculose et de progression de la tuberculose active chez les fumeurs peut aussi entraîner une prolifération rapide des groupes de nouveaux cas. Dans ce contexte, il peut s'avérer utile d'intégrer les initiatives de lutte contre le tabagisme et la consommation de substances mises de l'avant par différents programmes. Les programmes de lutte contre la tuberculose à Nunatsiavut (Terre-Neuve) et à Nunavik, au Québec - qui font tous deux face à des éclosions de tuberculose - ont récemment lancé des initiatives d'approche intégrant des messages de réduction des méfaits en lien avec la marijuana, élaborés en collaboration avec des jeunes de la communauté (communication personnelle, T. Buckle et le $D^{r}$ F. Bouchard, 4 octobre 2017).

Le tabagisme est l'un des rares facteurs de risque potentiellement réversibles pour la tuberculose, et touche plusieurs points dans I'histoire naturelle de la maladie. L'OMS et d'autres instances préconisent l'intégration des initiatives de lutte contre le tabagisme et la tuberculose $(3,6)$ organisées à l'échelon des cliniques et des programmes. Nous croyons qu'il existe de nombreuses possibilités de réaliser une telle intégration au Canada. Les initiatives de lutte contre le tabagisme peuvent aider à réduire la transmission locale et le nombre de nouveaux cas aussi bien dans les villes du Sud que dans celles du Nord.

\section{Déclaration des auteurs}

E.R. - Conceptualisation, Enquête, Rédaction - ébauche originale, rédaction - examen et révision, supervision T.L. - Conceptualisation, Enquête, Rédaction - ébauche originale, rédaction - examen et révision

Elizabeth Rea était la rédactrice invitée pour ce numéro du RMTC mais s'est abstenue de prendre part aux décisions éditoriales relatives à ce manuscrit. Les décisions ont été prises par la Dre Patricia Huston, rédactrice en chef.

\section{Conflit d'intérêt}

Aucun.

\section{Collaborateurs}

Adrian Sebastian - Supervision, Enquête

Bernard Lee - Enquête

\section{Remerciements}

Les auteurs tiennent à remercier les nombreuses personnes qui ont contribué à l'enquête sur les groupes, y compris les personnes atteintes de tuberculose active, le gestionnaire de bâtiment $A$. da Cunha, les infirmières de la tuberculose au Bureau de santé publique de Toronto, ainsi que le personnel du Laboratoire de tuberculose de Santé publique Ontario.

\section{Références}

1. Lin HH, Ezzati M, Murray M. Tobacco smoke, indoor air pollution and tuberculosis: a systematic review and meta-analysis. PLoS Med 2007 Jan;4(1):e20. https://doi. org/10.1371/journal.pmed.0040020. PubMed (https://www. ncbi.nlm.nih.gov/entrez/query.fcgi?cmd=Retrieve\&db=PubMe d\&list_uids=17227135\&dopt=Abstract).

2. Bates MN, Khalakdina A, Pai M, Chang L, Lessa F, Smith KR. Risk of tuberculosis from exposure to tobacco smoke: a systematic review and meta-analysis. Arch Intern Med 2007 Feb;167(4):335-42. Epub 2011 Feb 28. http://dx.doi. org/10.1001/archinte.167.4.335. PubMed (https://www.ncbi. $\mathrm{nlm}$.nih.gov/entrez/query.fcgi? $\mathrm{cmd}=$ Retrieve\&db=PubMed\&li st_uids=17325294\&dopt=Abstract).

3. World Health Organization. Tuberculosis \& Tobacco Final TB Fact Sheet. World Health Organization; November 2009 [Consulté le 2 nov 2017]. http://www.who.int/tobacco/ resources/publications/factsheet_tub_tob.pdf

4. Shang S, Ordway D, Henao-Tamayo M, Bai X, Oberley-Deegan $\mathrm{R}$, Shanley $\mathrm{C}$ et al. Cigarette smoke increases susceptibility to tuberculosis-evidence from in vivo and in vitro models. J Infect Dis 2011 May;203(9):1240-8. http://dx.doi. org/10.1093/infdis/jir009. PubMed (https://www.ncbi.nlm. nih.gov/entrez/query.fcgi?cmd=Retrieve\&db=PubMed\&lis t_uids=21357942\&dopt=Abstract).

5. O'Leary SM, Coleman MM, Chew WM, Morrow C, McLaughlin AM, Gleeson LE et al. Cigarette smoking impairs human pulmonary immunity to Mycobacterium tuberculosis. Am J Respir Crit Care Med 2014 Dec;190(12):1430-6. http://dx.doi. org/10.1164/rccm.201407-1385OC. PubMed (https://www. ncbi.nlm.nih.gov/entrez/query.fcgi?cmd=Retrieve\&db=PubMe d\&list_uids=25390734\&dopt=Abstract).

6. van Zyl-Smit RN, Brunet L, Pai M, Yew WW. The convergence of the global smoking, COPD, tuberculosis, HIV, and respiratory infection epidemics. Infect Dis Clin North Am 2010 Sep;24(3):693-703. http://dx.doi.org/10.1016/j. idc.2010.04.012. PubMed (https://www.ncbi.nlm.nih. gov/entrez/query.fcgi?cmd=Retrieve\&db=PubMed\&list_ uids=20674799\&dopt=Abstract).

7. Lin HH, Ezzati M, Chang HY, Murray M. Association between tobacco smoking and active tuberculosis in Taiwan: prospective cohort study. Am J Respir Crit Care Med 2009 Sep;180(5):475-80. http://dx.doi.org/10.1164/ rccm.200904-05490C. PubMed (https://www.ncbi.nlm. nih.gov/entrez/query.fcgi? $\mathrm{cmd}=$ Retrieve $\& \mathrm{db}=$ PubMed\&lis t_uids=19542475\&dopt=Abstract).

8. Yen YF, Yen MY, Lin YS, Lin YP, Shih HC, Li LH et al. Smoking increases risk of recurrence after successful anti-tuberculosis treatment: a population-based study. Int J Tuberc Lung Dis 2014 Apr;18(4):492-8. http://dx.doi.org/10.5588/ ijtld.13.0694. PubMed (https://www.ncbi.nlm.nih.gov/ entrez/query.fcgi?cmd=Retrieve\&db=PubMed\&list_ uids $=24670708 \&$ dopt $=$ Abstract).

9. Gajalakshmi V, Peto R, Kanaka TS, Jha P. Smoking and mortality from tuberculosis and other diseases in India: retrospective study of 43000 adult male deaths and 35000 
controls. Lancet 2003 Aug;362(9383):507-15. http://dx.doi. org/10.1016/S0140-6736(03)14109-8. PubMed (https://www. ncbi.nlm.nih.gov/entrez/query.fcgi?cmd=Retrieve\&db=PubMe d\&list_uids=12932381\&dopt=Abstract).

10. Lindsay RP, Shin SS, Garfein RS, Rusch ML, Novotny TE. The Association between active and passive smoking and latent tuberculosis infection in adults and children in the united states: results from NHANES. PLoS One 2014 Mar;9(3):e93137. https://doi.org/10.1371/journal.pone.0093137.

PubMed (https://www.ncbi.nlm.nih.gov/entrez/query.fcgi?cmd $=$ Retrieve \&db=PubMed\&list_uids=24664240\&dopt=Abstract).

11. Patra J, Bhatia M, Suraweera W, Morris SK, Patra C, Gupta PC et al. Exposure to second-hand smoke and the risk of tuberculosis in children and adults: a systematic review and meta-analysis of 18 observational studies. PLoS Med 2015 Jun;12(6):e1001835. http://dx.doi.org/10.1371/ journal.pmed.1001835. PubMed (https://www.ncbi.nlm. nih.gov/entrez/query.fcgi? cmd=Retrieve \&db=PubMed\&lis t_uids=26035557\&dopt=Abstract).

12. Huang CC, Tchetgen ET, Becerra MC, Cohen T, Galea J, Calderon $\mathrm{R}$ et al. Cigarette smoking among tuberculosis patients increases risk of transmission to child contacts. Int J Tuberc Lung Dis 2014 Nov;18(11):1285-91. http://dx. doi. org/10.5588/ijtld.14.0309. PubMed (https://www.ncbi.nlm. nih.gov/entrez/query.fcgi? $\mathrm{cmd}=$ Retrieve $\& \mathrm{db}=$ PubMed\&lis t_uids=25299859\&dopt=Abstract).

13. Jha P, Jacob B, Gajalakshmi V, Gupta PC, Dhingra N, Kumar $R$ et al.; RGI-CGHR Investigators. A nationally representative case-control study of smoking and death in India. N Engl J Med 2008 Mar;358(11):1137-47. http://dx.doi.org/10.1056/ NEJMsa0707719. PubMed (https://www.ncbi.nlm.nih. gov/entrez/query.fcgi?cmd=Retrieve\&db=PubMed\&list_ uids $=18272886 \&$ dopt $=$ Abstract) .

14. Menzies D, editor. Normes canadiennes pour la lutte antituberculeuse 7ième édition 2014. Société canadienne de thoracologie, Association pulmonaire du Canada et l'AGence de la sant/ publique du Canada, 2014. https://cts.lung.ca/fr/ node/91?

15. Réseau pancanadien de santé publique. Orientations pour les programmes de prévention et de contrôle de la tuberculose au Canada [Consulté le 24 jan 2018. http://www.phn-rsp.ca/ pubs/gtbpcp-oppctbc/pdf/Orientations-de-prevention-d e-la-tuberculose-fra.pdf

16. Statistique Canada. Fumeurs, selon le sexe, provinces et les territoires (Pourcentage). Statistique Canada; 2016 [Consulté le 26 oct 2017]. http://statcan.gc.ca/tables-tableaux/sum-som/ 102/cst01/health74b-fra.htm

17. Toronto SH. The Health of Toronto's Homeless Population. Finding Home: Policy Options for Addressing Homelessness in Canada. Canadian Observatory on Homelessness. 2009 [Consulté le 29 déc 2017]. http://homelesshub.ca/resource/2 2-health-toronto\%E2\%80\%99s-homeless-population
18. Statistique Canada. Tableau 577-0008 - Enquête auprès des peoples autochtones, statut sur l'usage du tabac, selon le groupe d'âge et sexe, population inuite âgés de 15 ans et plus, Canada et Inuit Nunangat. CANSIM (base de données). [Consulté le 3 janv 2018]. http://www5.statcan.gc.ca/cansim/ a26? lang=fra\&retrLang $=$ fra\&id $=5770008 \&$ pattern $=\&$ stByVal =1\&p1=1\&p2=31\&tabMode=dataTable \&csid=

19. Toronto Public Health. Communicable Disease Surveillance Reports. 2016 [Consulté le 24 jan 2018]. https://www.toronto. $\mathrm{ca} /$ community-people/health-wellness-care/health-inspectionsmonitoring/communicable-disease-surveillance-reports/

20. Statistique Canada. 2017. Toronto, CDR [Profil du recensement], Ontario et Canada [Country] (Tableau). Recensement de 2016. Statistique Canada No 98-316X2016001 au catalogue. Ottawa. Mise à jour le octobre 2017 [Consulté le 1 nov 2017]. http://www12.statcan.gc.ca/ census-recensement/2016/dp-pd/prof/index.cfm?Lang=F

21. Oeltmann JE, Oren E, Haddad MB, Lake L, Harrington TA, ljaz K et al. Tuberculosis outbreak in marijuana users, Seattle, Washington, 2004. Emerg Infect Dis 2006 Jul;12(7):1156-9. http://dx.doi.org/10.3201/eid1207.051436. PubMed (https:// www.ncbi.nlm.nih.gov/entrez/query.fcgi?cmd=Retrieve\&db=Pu bMed\&list_uids=16836841\&dopt=Abstract).

22. Munckhof WJ, Konstantinos A, Wamsley M, Mortlock M, Gilpin C. A cluster of tuberculosis associated with use of a marijuana water pipe. Int J Tuberc Lung Dis 2003 Sep;7(9):860-5.

PubMed (https://www.ncbi.nlm.nih.gov/entrez/query.fcgi?cmd $=$ Retrieve\&db=PubMed\&list_uids=12971670\&dopt=Abstract).

23. Leonhardt KK, Gentile F, Gilbert BP, Aiken M. A cluster of tuberculosis among crack house contacts in San Mateo County, California. Am J Public Health 1994 Nov;84(11):1834-6. http:// dx.doi.org/10.2105/AJPH.84.11.1834. PubMed (https://www. ncbi.nlm.nih.gov/entrez/query.fcgi?cmd=Retrieve\&db=PubMe d\&list_uids=7977929\&dopt=Abstract).

24. Gallant V, Duvvuri V, McGuire M. La tuberculose au Canada - Résumé 2015. Relevé des maladies transmissibles au Canada. 2017;43(3/4):85-91. https://www.canada.ca/fr/ sante-publique/services/rapports-publications/releve-maladie s-transmissibles-canada-rmtc/numero-mensuel/2017-43/ rmtc-volume-43-3-4-2-mars-2017/rmtc-volume-43-3-4-2mars-2017-tuberculose-1.html

25. Bougie $E$, Kohen D. Smoking prevalence among Inuit in Canada. Health Rep 2017 Feb;28(2):10-4. PubMed (https:// www.ncbi.nlm.nih.gov/pubmed/28198984)

26. Vachon J, Gallant V, Siu W. La tuberculose au Canada, 2016. Relevé des maladies transmissibles au Canada. 2018;44(3/4):85-91. https://www.canada.ca/fr/ sante-publique/services/rapports-publications/releve-maladie s-transmissibles-canada-rmtc/numero-mensuel/2018-44/ numero-3-4-1-mars-2018/article-1-tuberculose-2016.html 